\title{
Giuliano Milani
}

\section{La fedeltà di Dante a Moroello}

\section{L'epistola IV dalla prospettiva del destinatario}

\begin{abstract}
L'articolo rilegge l'epistola IV dando particolare importanza al suo destinatario, il marchese Moroello Malaspina. Dopo aver riconfermato sulla base degli studi storici e genealogici l'identificazione di questi con Moroello di Manfredi di Giovagallo, si indaga la natura della relazione che unì questo signore a Dante, sottolineandone tre elementi: la continuità (solo brevemente interrotta) della militanza sullo stesso fronte politico; la specificità del carattere "cortese" di questa relazione; la maggiore compatibilità di questo rapporto con il progetto imperiale che si costruì intorno a Enrico VII che con quello anti-imperiale fiorentino.
\end{abstract}

The article reads the fourth epistle giving particular importance to its addressee, the Marquis Moroello Malaspina. It reaffirms the identification of the latter with Moroello di Manfredi di Giovagallo on the basis of historical and genealogical research. It explores the relationship between this lord and Dante stressing three elements: its political coherence (only briefly interrupted); its peculiar "court" character; its better compatibility with the imperial project built around Henry VII than with the Guelph one led by Florence.

Parole chiave: Dante, epistola IV, Moroello Malaspina, Cino da Pistoia, Lunigiana, impero, Enrico VII, uomini di corte, giustizia, autobiografia.

Benché aggiunta dal copista, l'intestazione costituisce uno dei pochi dati su cui possiamo fondare l'analisi del misterioso testo dell'epistola IV. ${ }^{1}$ È questo paratesto, infatti, a dirci che la lettera è indirizzata da Dante al marchese Moroello Malspina. Nonostante negli ultimi anni sia stato fatto un notevole lavoro esegetico, ${ }^{2}$ tutto il resto rimane controverso: se la lettera sia stata scritta a ridosso dell'incontro di Dante con i Malaspina, testimoniato dalla cosiddetta "pace

1 BAV, Vat. Pal. Lat. 1729, f. 60; Ep. IV, 1: «[Scribit Dantes domino Moroello marchioni Malaspine]».

2 Per una panoramica degli studi recenti e delle soluzioni apportate negli ultimi anni ai principali problemi, Tonelli, La canzone montanina.

Giuliano Milani, Université Paris Est Marne-la-Vallée

Ә Open Access. (C) 2020 Giuliano Milani, published by De Gruyter. (c) BY This work is licensed under the Creative Commons Attribution 4.0 International License (CC BY 4.0). 
di Castelnuovo" (ottobre 1306), oppure in anni più lontani, se faccia riferimento a un vero innamoramento del suo autore o sia piuttosto un testo allegorico; se, infine, accompagnasse in origine la canzone Amor, da che convien pur ch'io mi doglia detta "montanina" o un qualche altro testo.

L'esperimento che si tenterà in queste pagine consiste nel provare a utilizzare l'intestazione (l'indirizzo a Moroello) per avanzare di qualche passo (non molti) nella risposta alle questioni più incerte, a cominciare dalla datazione. Per svolgerlo cominceremo da alcuni chiarimenti sul profilo politico di Moroello (2), proseguendo con l'analisi degli argomenti proposti per la datazione più bassa (circa 1311-1313) (3), quindi di quella più alta (circa 1306-1309) (4). Infine proveremo a valorizzare, sulla base dei risultati di queste analisi, la relazione di Dante con Moroello (5) nel quadro più ampio (6) del percorso biografico e autobiografico del poeta. Prima di tutto ciò, tuttavia, sarà necessario soffermarsi ancora una volta sull'identità del destinatario.

\section{Quale Moroello?}

Anche dando per certo che il destinatario sia Moroello Malaspina, infatti, i problemi relativi alla sua identificazione non si esauriscono completamente, perché di Moroelli, tra i Malaspina, ce n'erano parecchi. Com'era tipico dei lignaggi aristocratici medievali, anche in questa famiglia distaccatasi attorno alla metà del secolo XII da una delle più antiche stirpi dell'aristocrazia italiana, quella dei marchesi Obertenghi, originati da Oberto I (morto nel 973), signore della marca ianuensis, delle contee di Luni, di Genova e Tortona, i nomi dello stock onomastico familiare si ripetevano spesso, seguendo talvolta precise regole di trasmissione. All'epoca che ci interessa, il nome Moroello si ritrova in particolare nel ramo detto dello "spino secco", originato da Corrado di Obizzo di Obizzo (definito in Purgatorio «l'antico»). ${ }^{3}$

Tra i Malaspina dello "spino secco" ci sono infatti almeno tre Moroelli. Il primo è il marchese Moroello, figlio di Corrado stesso, signore di Mulazzo e padre di Barnabò, vescovo di Luni, di Elena e di Franceschino. La sua morte, avvenuta al più tardi nel 1284 (o 1285) in Corsica, impedisce tuttavia di ritenere che sia il Moroello dantesco. Il secondo è il figlio di suo figlio Franceschino, attestato fino al 1370. In questo caso, tuttavia, è l'eccessiva giovinezza a escluderlo dal novero dei candidati, dal momento che nel 1321 era ancora minorenne

3 Il ramo dello "spino fiorito" raccoglieva invece i discendenti di Obizzino, figlio di un cugino di Corrado "l'antico". Tavola genealogica in Fiori, I Malaspina, p. 324. 
e affidato alla tutela di Castruccio Castracani. ${ }^{4}$ Per la stessa ragione, anche se con appena minore certezza, si è portati di escludere un terzo Moroello, suo cugino di terzo grado, figlio di Obizzino di Federico, signore di Villafranca, che appare minore e sotto tutela della madre Tobia Spinola in un documento del 1301 e che muore prima del $1321 .{ }^{5}$ Come si vedrà, se in teoria questo Moroello potrebbe essere un (giovanissimo) protettore di Dante, gli argomenti a suo favore non sono forti come quelli a favore del più famoso Morello di Giovagallo. Il fatto poi che i genealogisti, sulla base della documentazione, non attribuiscano a Moroello di Villafranca il titolo marchionale che hanno, invece, i suoi fratelli maggiori, è un motivo in più per pensare che l'intitolazione dell'epistola non si riferisca a lui. ${ }^{6}$ Da eliminare con certezza è infine un altro candidato avanzato dall'erudizione ottocentesca, il presunto Moroello di Alberto di Corrado "di Val di Trebbia". ${ }^{7}$ La ricerca ha dimostrato che questo personaggio attestato da un documento del 1322 è semplicemente un abitante di Mulazzo che non ha nulla a che vedere con la famiglia Malaspina. ${ }^{8}$

Benché dunque un minimo di incertezza continui a sussistere, la genealogia della famiglia Malaspina porta a credere dunque con una notevole verosimiglianza che il destinatario dell'epistola IV sia il marchese Moroello di Manfredi di Corrado "l'antico", signore di Giovagallo, nato verso la fine degli anni 1260 e destinato a morire nel $1315 .{ }^{9}$ Convergono verso di lui anche gli indizi tratti dai testi danteschi. È lui che Dante cita nell'Inferno, alludendovi con la profezia di Vanni Fucci che ricorda la sua conquista di Pistoia (1306) definendolo «vapor di Val di Magra» (Inf. XXIV, 145). È sempre lui che, nel Purgatorio, fa capolino dietro la lode che papa Adriano IV fa di Alagia Fieschi, che era stata sua moglie (Purg. XIX, 142-145). Anche, ma non solo, alla luce di questa corrispondenza tra documenti malaspiniani e danteschi, la stragrande maggioranza degli studiosi, soprattutto nell'ultimo secolo, lo hanno identificato con colui che nei documenti della pace con il vescovo di Luni dell'ottobre 1306, insieme ai cugini Franceschino e Corrado, è rappresentato da Dante. ${ }^{10}$ Alla luce di tutto ciò sembra un'ipotesi davvero antieconomica ritenere che sia altra persona il dedicatario del sonetto di Cino da Pistoia Cercando di trovar minera in oro a cui Dante ri-

4 Fa il punto Bianchi, La poesia d'amore, p. 51.

5 Saffioti Bernardi, Malaspina, Moroello.

6 Questo elemento non mi pare sufficientemente sottolineato dagli studi danteschi.

7 Branchi, Storia della Lunigiana feudale, I, pp. 178-189.

8 Fiori, I Malaspina di Pregola, p. 265.

9 Salvatori, Malaspina, Moroello.

$10 C D D$, p. 237, dove nella nota storica, redatta da chi scrive, andrà corretta un'inesattezza: ove si legge «fratello» all'ultima riga, bisognerà leggere «figlio». 
sponde, secondo le intitolazioni della tradizione, "in nome del marchese Malaspina" col sonetto Degno fa voi trovare ogni tesoro (Rime CXIII) nel contesto di una più ampia corrispondenza che permette di inserire l'epistola IV in un piccolo corpus dantesco-ciniano-malispiniano composto da sette testi: cinque sonetti in volgare e due epistole latine (la III, a Cino, e la IV), sul quale ritorneremo. ${ }^{11}$

\section{Un percorso politico comune brevemente interrotto}

Il principale ostacolo che nel corso della seconda metà dell'Ottocento ha impedito agli studiosi di riconoscere questo Moroello come l'ospite e il protettore di Dante, generando la necessità di trovare (e, come si è visto, talvolta, di inventare) omonimi tra i rami del lignaggio marchionale, è che il marchese e il poeta militarono su due fronti opposti nel conflitto che segnò la Toscana nei primi anni del Trecento. Come era stato possibile che Dante, schierato con i Bianchi a Firenze negli anni a cavallo del secolo, dichiarato colpevole di corruzione e bandito dai Neri nel 1302, impegnatosi dal punto di vista militare e diplomatico con gli altri esuli tra 1302 (convegno di San Godenzo) e il 1304 (probabile data dell'epistola I), avesse coltivato relazioni di amicizia con il capitano generale dell'esercito nero formato da Lucca e Firenze, che nel 1302 aveva combattuto contro Pistoia conquistando la fortezza di Serravalle e che nel 1306, dopo essere stato eletto capitano della taglia guelfa, aveva conquistato la stessa Pistoia, rovesciando in favore dei Neri fiorentini, l'equilibrio politico regionale?

Ė interessante notare che questo problema non assillò affatto i biografi antichi, i quali non sembrano percepire la contraddizione. Boccaccio, per esempio, nelle sue due versioni del Trattatello, pur concedendo ampio spazio a Moroello Malaspina (del quale afferma non solo che fu ospite del poeta, ma anche suggeritore della "ripresa" della scrittura della Commedia, nonché, probabilmente sulla base della cosiddetta Epistola di Ilaro, dedicatario del Purgatorio), ${ }^{12}$ non accenna mai al fatto che militasse su un fronte diverso e anzi, nelle Esposizioni lo definisce «uomo intendente e in singolarità suo [di Dante] amico»..$^{13}$

11 Barnes, Moroello.

12 Boccaccio, Trattatello (I ${ }^{\mathrm{a}}$ red.), XIII, 181-182, pp. 99-100; 104, 106-107, 112; (II ${ }^{\mathrm{a}}$ red.), p. 130, 146, 148, 172.

13 Boccaccio, Esposizioni, pp. VIII, XXIII. 
Tra le ragioni di questa sottovalutazione del problema dell'incompatibilità politica è forse il fatto che, come emerge dalla ricerca più recente, presto Dante e Moroello si trovarono a militare dalla stessa parte, quella dei sostenitori italiani di Enrico VII. Anche in questo caso l'erudizione ottocentesca, a causa dei medesimi pregiudizi sull'orientamento politico a cui si faceva riferimento in apertura, ha contribuito non poco alla confusione, storpiando notizie cronistiche e finendo per ipotizzare un passaggio del marchese all'esercito guelfo negli anni dello scontro tra Enrico e Firenze. ${ }^{14}$ Ma le cose non stanno così. Come ha chiarito Enrica Salvatori sciogliendo definitivamente i dubbi, Moroello, benché capitano dei Neri di Lucca alleati a Firenze fino al 1306-1307, finì per allearsi con Enrico VII sin dal suo arrivo in Italia. ${ }^{15}$ Egli incontrò l'imperatore già nel dicembre 1310 a Vercelli ${ }^{16}$ e lo seguì a Milano, dove assistette alle prime pacificazioni delle città e ottenne la conferma dei suoi possedimenti. ${ }^{17}$ Insieme ai suoi congiunti contribuì all'impresa enriciana versando 100 fiorini d'oro per quanto spettava ai suoi possessi "in Lombardia", ${ }^{18}$ e partecipando nel giugno 1311 all'assedio di Brescia, città della quale, dopo la conquista, nel settembre di quell'anno, divenne vicario imperiale. ${ }^{19}$ La stessa autrice sottolinea come «negli anni successivi il M. mantenne, al pari degli altri esponenti della sua famiglia, una posizione di guelfismo moderato che non si opponeva frontalmente ai disegni imperiali, ma anzi li sosteneva talvolta anche militarmente» e che «i fattori ambientali e politici che cementarono il legame [con Dante] non vennero meno anche dopo il 1307, anzi si prolungarono fino alla morte dello stesso Malaspina», cioè fino al $1315 .^{20} \mathrm{Si}$ tratta di dati importanti, credo, le cui conseguenze non sono state ancora valutate pienamente negli studi danteschi. ${ }^{21}$

A questa vicinanza politica tra Dante e Moroello negli anni successivi al loro primo contatto nel 1306 si potrebbe aggiungere un'analoga sintonia negli anni precedenti al 1302. Tralasciando, in attesa di nuovi chiarimenti, la partecipazione di Moroello Malaspina alla battaglia di Campaldino, sostenuta da alcuni eruditi ma per nulla provata, si può osservare che certamente nel 1297 Moroello fu capitano dell'esercito bolognese nella guerra contro Azzo d'Este per

14 Gerini, Memorie storiche, II, p. 43-45. Branchi, Storia della Lunigiana, I, pp. 505.

15 Salvatori, Malaspina, Moroello.

16 Henrici VII Constitutiones, pp. 445, 449-450.

17 Henrici VII Constitutiones, pp. 453, 459, 500-502.

18 Henrici VII Constitutiones, p. 510.

19 Henrici VII Constitutiones, pp. 586.

20 Salvatori, Malaspina, Moroello.

21 Continua a esprimere perplessità sulla compatibilità politica tra Dante e Moroello Graziosi, Dante a Cino: e prima di lei Baron, La conoscenza della Commedia. Più prudente Casadei, Dante oltre la Commedia, p. 133. 
divenire poi, a partire dal novembre di quello stesso anno, podestà di Bologna. ${ }^{22}$ Nel corso di questo periodo Firenze era ancora un'alleata strategica della città felsinea e non c'è ragione di credere che Dante, che aveva partecipato subito prima al governo fiorentino e che vi avrebbe partecipato subito dopo, non condividesse pienamente questa alleanza. Nel 1299 Moroello, proseguendo la sua brillante carriera di condottiero militare, divenne capitano generale dell'esercito che Matteo Visconti lanciava insieme a Bologna, Parma, Piacenza e Verona contro il marchese Giovanni di Monferrato. Nella Firenze ormai in procinto di dividersi tra Bianchi e Neri, erano i primi (e, tra loro, Dante) a sostenere le parti del signore di Milano e con lui del suo capitano. ${ }^{23}$

L'incompatibilità politica tra Moroello e Dante durò dunque poco. Dal punto di vista di Moroello si trattò di quattro anni: dal 1302 (data dell'inizio del comando di Moroello dell'esercito nero fiorentino) al 1306 (quando insieme ai suoi parenti Francesco e Corrado fece di Dante il suo rappresentante, e dunque o il poeta o il marchese avevano mosso un passo l'uno nella direzione dell'altro). Ancora meno durò tale incompatibilità se si considera il punto di vista di Dante. Se infatti si prende per buona la versione che volle esprimere nella Commedia, ${ }^{24}$ i contatti con il signore lunigianese furono improbabili solo dal bando del 1302 al distacco dalla parte dei Bianchi, quella che avrebbe definito «compagnia malvagia e scempia» (Par. XVII, 62), databile, a seconda delle opinioni, tra 1303 e 1304. In ogni caso, l'incompatibilità costituì una parentesi brevissima nell'ambito di una lunga militanza sullo stesso fronte, o meglio, sullo stesso lato di una frontiera mutevole.

I casi paralleli di Dante e di Moroello mostrano infatti la difficoltà di considerare le parti di questi anni (Guelfi/Ghibellini e, ancora di più, Bianchi/Neri) come schieramenti permanenti, ereditati e dotati di uno spessore ideologico capace di mantenerli invariati. Le parti che si combattevano in Italia al contrario cambiavano spesso, soprattutto per effetto del mutare delle relazioni sovraregionali come quelle triangolari tra la Lombardia (in cui agivano i Visconti, $\mathrm{i}$ Della Torre e il marchese di Monferrato), Bologna con la Romagna e, infine, la Toscana delle città e dei signori rurali. Quanti, come Dante e Moroello, per ragioni diverse, si muovevano su uno scacchiere sovra-cittadino, dovevano, di volta in volta, adattarsi. L'impresa di Enrico VII, coinvolgendo tutte le realtà politiche nello stesso conflitto com'era avvenuto all'epoca di Federico II, avreb-

22 Fa il punto Bortoluzzi, I rapporti diplomatici.

23 Milani, Dante politico fiorentino.

24 Indizio, La profezia di Cacciaguida. 
be dato provvisoriamente un certo ordine a questo magma che nel primo decennio del secolo aveva tuttavia raggiunto il massimo livello di mutevolezza. ${ }^{25}$

A tutto ciò occorre infine aggiungere il fatto che, a differenza di quanto è stato enfatizzato negli ultimi anni, non è affatto detto che questa incompatibilità fosse un motivo dirimente a impedire il contatto tra un navigato signore rurale e un fiorentino assai meno noto che sino a dieci anni prima non aveva svolto alcuna attività politica.

Pure volendo considerare questa differenza come dirimente, va detto che se queste considerazioni restringono con nettezza il periodo in cui l'epistola non poté essere scritta (1302-1303), e dunque liberano definitivamente dai dubbi che afflissero gli studiosi ottocenteschi circa l'identità del destinatario, esse non facilitano il compito di datarla. Non per questo mi pare necessario ampliare ulteriormente il ventaglio delle datazioni finora ipotizzate, che, pur nelle loro variazioni, possono essere ricondotte a due momenti: uno che la colloca nei pressi della pace di Castelnuovo, l'altro che la spinge verso gli anni di Enrico VII. ${ }^{26}$ La ragione per cui non proveremo nemmeno ad anticipare la datazione di questa epistola a prima del 1306 o a posticiparla rispetto al 1313 è che la compatibilità politica tra mittente e destinatario è un elemento necessario ma non sufficiente. Per formulare all'interno del periodo possibile una datazione più precisa occorrono elementi positivi. Proviamo a valutare questi elementi esaminando più da vicino le due datazioni che sono state proposte.

\section{Scrivere a Moroello tra 1311 e 1313: Dante uomo d'impero}

Cominciamo dalla datazione più bassa, quella formulata sulla base di argomenti diversi da Pascoli e Torraca e oggi sostenuta da Claudia Villa. ${ }^{27}$ Il fatto che le

25 Cfr. l'articolo di Paolo Grillo in questo volume, ma anche Bortoluzzi, I rapporti diplomatici e Milani, I contesti politici.

26 Ep. (Villa) p. 1531: «quindi la datazione del biglietto, in mancanza di indicazioni, poiché la lettera è incompleta, ha suscitato non pochi interventi, oscillando fra il tempo in cui un documento conferma la presenza di Dante a Sarzana e poi a Castelnuovo di Magra, in veste di procuratore dei cugini Malaspina (Moroello, Corradino e Franceschino) nella conclusione della pace con il vescovo di Luni, e il suo soggiorno nell'alto Valdarno, certificato dalle epistole politiche datate alla primavera del 1311». Ep. (Baglio), p. 90: «per la datazione dell'epistola si possono proporre il biennio 1307-1308 o meglio quello 1308-1309 [con citazione di Fenzi, Ancora sulla Epistola e Pasquini, Un crocevia]». Baglio non condivide l'ipotesi di Villa per cui la lettera andrebbe spostata cronologicamente intorno al 1311 (p. 92).

27 Pascoli, Mirabile visione; Torraca, rec. a Zenatti, Dante e Firenze, pp. 139-160. Villa, Un oracolo e una ragazza. 
prove, già presentate in più sedi, si ritrovino nel suo saggio pubblicato in questo stesso volume esime dal riportarle analiticamente. Punto di partenza è l'argomento, ex silentio ma pesante, per cui la relazione tra questa epistola e la canzone Amor, da che convien pur ch'io mi doglia non ha alcun fondamento nella tradizione manoscritta. Partendo da questo fatto e accogliendo pienamente la vicinanza di Moroello a Enrico VII negli anni 1310-1313 - che, come si è visto difficilmente si fa strada negli studi danteschi, Villa osserva che alcuni dei termini chiave del testo dell'epistola IV fanno pensare che accompagnasse piuttosto un testo politico. "Oraculum” ( $E p$. IV, 1), che designa la lettera stessa, rinvierebbe a una «espressione solenne della volontà imperiale» ${ }^{28}$ e più in generale è termine che il Somnium Scipionis («testo di formidabile riflessione politica e civile») definisce come un messaggio ricevuto in sonno da un'autorità relativamente a ciò che succederà o meno e a ciò che va fatto o non fatto. ${ }^{29} \mathrm{Il}$ «magnificientia vestra» del primo paragrafo viene accostato al termine usato per Cangrande nell'epistola XIII, sottolineando che entrambi erano vicari imperiali. ${ }^{30}$ Gli «officia» che Dante scrive di aver esercitato destando l'ammirazione del suo destinatario sembrano doveri pubblici e dunque «difficilmente riconducibili a privati esercizi poetici». ${ }^{31}$ Infine - soprattutto - la "curia" «in seguito ricordata con nostalgia», a cui Dante fa riferimento, non sarebbe «una qualsiasi corte feudale attiva nel $1306 »$; $^{32}$ essa potrebbe invece costituire un riferimento alla curia imperiale di Enrico VII che Dante e Moroello avrebbero raggiunto a Pisa nel 1311. In questo senso gli “officia libertatis” potrebbero essere gli incarichi nel corso dei quali Dante avrebbe formulato le riflessioni sul potere imperiale di cui si trova la traccia più rilevante nella Monarchia. L'oracolo in cui la lettera costituirebbe, farebbe inoltre pensare all'oracolo pronunciato da Beatrice nel canto XXXIII del Purgatorio. Della fine di questa cantica, conclude la Villa, l'epistola IV sarebbe "sinopia", ovvero una sorta di disegno preparatorio.

Tralasciando per il momento questi argomenti su cui si tornerà tra breve, vale la pena di sottolineare che l'ipotesi che l'epistola IV sia stata scritta intorno al 1312 presuppone almeno tre fatti: 1) che Dante sia effettivamente stato a Pisa o altrove presso la curia di Enrico VII; 2) che in quel contesto abbia esercitato ufficialmente qualche ufficio per conto dell'imperatore; 3) che di lì sia passato anche Moroello, osservandolo "con ammirazione”.

28 Villa, Tempi dell'epistolario dantesco.

29 Ep. (Villa), p. 1533.

30 Villa, Tempi dell'epistolario dantesco, p. 238.

31 Villa, Tempi dell'epistolario dantesco, p. 239.

32 Ep. (Villa), p. 1533. 
Non c'è dubbio che questi fatti, benché non documentati, siano del tutto plausibili, al punto da essere stati proposti, separatamente o insieme, anche da altri studiosi. ${ }^{33}$ Più difficile è tuttavia inserire in questo contesto cronologico suggerito da elementi che nel testo dell'epistola sono per certi versi accessori, degli elementi assai più sostanziali: il fatto narrato e il suo destinatario.

All'analisi letterale, anche prescindendo dalla relazione con la canzone “montanina”, l'oggetto dell'epistola IV è infatti, senza possibilità di dubbio, una violenta trasformazione, avvenuta in quel momento nel suo autore, innescata dal ritorno di Amore, che mette fine per Dante a una fase scandita dal proposito di astenersi dalle donne e dai loro canti. È possibile immaginare che Dante, all'altezza del 1312, e dunque a Inferno e Purgatorio probabilmente conclusi, anche se forse non completamente rivisti, dica che questo ritorno di Amore sta avvenendo in quel momento? Non è troppo tardi? Non si rischia di sovrapporre Dante-personaggio, del quale probabilmente proprio intorno al $1312 \mathrm{i}$ primissimi lettori vennero a sapere che aveva ritrovato Beatrice nel paradiso terrestre, col Dante-poeta che da qualcosa come cinque anni doveva aver deciso di riprendere in mano il progetto di cantarla e che lo aveva fatto in un'opera in cui quella decisione era stata retrodatata di altri sette anni, alla primavera 1300 ? Le perplessità si fanno più intense se si considera il passo iniziale dell'Epistola IV, quello in cui Dante motiva la necessità di comunicare questo fatto al Marchese al fine di evitare il diffondersi di false opinioni che rischiano di farlo passare per negligente quando invece non ha più scelta. Quale negligenza verso Moroello si potrebbe rimproverare a Dante all'indomani della scrittura delle epistole politiche "arrighiane" e delle prime due cantiche della Commedia se non, addirittura, della Monarchia (se si accetta una datazione alta di quest'opera), se si propende per una redazione dell'epistola tutta svoltasi nel contesto di una relazione tra i due ormai interna al contesto della curia imperiale?

Quanto al destinatario, benché, come si è detto e come si ridirà, sia possibilissimo che a quell'altezza il suo schieramento fosse lo stesso di quello in cui militava il poeta, non mi sembra attestato che tra 1311 e 1313 Dante intrattenga con lui una relazione di prossimità tale da potersi definire come "suo servo". Certo, è molto probabile che ci siano stati nuovi contatti tra i due. Lo dimostra non solo il fatto, già ricordato, che Moroello, una volta fattosi filoimperiale, non si staccò mai davvero dalla fedeltà a Enrico, ma anche la possibilità, ricavabile dalla cosiddetta lettera di Ilaro - ritenuta plausibile anche da chi considera quel testo un falso ${ }^{34}$ - di un passaggio di Dante in Lunigiana tra l'agosto 1314 e

33 Dante, Enrico VII e Pisa, ma vedi le critiche di Pellegrini, Dante: biografia.

34 Inglese, Vita di Dante, p. 128. 
l'aprile $1315 .{ }^{35}$ Tali fatti tuttavia non offrono conferme all'esistenza di un rapporto tra i due stretto come quello presupposto dall'epistola.

Certo, se si dimostrasse che la lettera di Ilaro è vera o se uno degli altri presupposti ricordati (in particolare il soggiorno di Dante e/o Moroello a Pisa) ricevesse più conferme di quante non ne ha attualmente, questa ipotesi di datazione assumerebbe un peso più rilevante. In attesa di tali sviluppi, tuttavia, il tenore dell'epistola porta a mantenere la prudenza e a rivalutare la datazione tradizionale.

\section{Scrivere a Moroello tra 1306 e 1310: Dante uomo di corte}

Osservare l'epistola IV concentrando l'attenzione sul suo destinatario fa infatti emergere altri elementi che spingono a riportare la datazione dagli anni del viaggio italiano di Enrico VII a quelli precedenti, gli anni aperti dall'arrivo di Dante in Lunigiana del 1306.

Partiamo dalla “curia” evocata nell'epistola. Diversamente da quanto sostiene Villa mi sembra che tale termine sia il più adatto a indicare la corte nei due sensi, spesso sovrapposti (assemblea di fedeli e tribunale), di un signore come Moroello. Per i Malaspina il termine è attestato nella documentazione, sin dal secolo XII. ${ }^{36}$ Concordo invece pienamente con Villa (e in questo anche con Marco Santagata) ${ }^{37}$ nel ritenere che questo termine, ben traducibile con l'italiano "corte", non sia riferibile necessariamente a un singolo castello. ${ }^{38}$ La curia di Moroello si spostava ed è dunque possibile che Dante, parlando della curia che poi ha rimpianto, faccia riferimento, più che a un luogo, a un gruppo di persone che assistevano il signore, un gruppo che tra il 1306 e il 1309 si spostò dall'assedio di Pistoia alla stessa Pistoia, quindi in Lunigiana, forse a Giovagal-

35 Bellomo, Il sorriso di Ilaro.

36 Lo si vede per esempio in un patto tra Genova e i Malaspina stipulato dopo il 1130 edito in Olivieri, Serie dei consoli, p. 320: «et si iam dicti marchiones reclamauerint se ianuensibus consulibus de aliquo suo uasallo nostre ciuitatis et ipse noluerit eis facere rationem et recipere in curia marchionum consules ianuenses constringent eum ad faciendam et ad recipiendam rationem in curia predictorum marchionum». Anche il termine "magnificentia vestra" è in qualche modo confermato dalla documentazione coeva: un documento stipulato da Moroello nel 1302 citato da Gerini, Memorie storiche, II, p. 39 lo definisce "Magnificus vir dominus Moroellus marchio Malaspine".

37 Santagata, Dante, p. 192.

38 Villa, Un oracolo e una ragazza. 
lo, forse a Verrucola, quindi nel 1310 a Vercelli, dove raggiunse l'altra curia, quella dell'imperatore.

Come si è visto, giocando sull'ossimoro di servitù e libertà che pervade tutta la lettera Dante afferma di aver svolto presso questa curia, sotto lo sguardo ammirato del suo signore, degli "officia libertatis". ${ }^{39}$ Come dimostrano le oscillazioni dei traduttori, il primo termine di questo sintagma indica ad un tempo dei doveri e degli uffici. Se nel 1312 questo termine avrebbe potuto riferirsi, come ha ipotizzato la Villa a un lavoro svolto per Enrico VII, di cosa si poteva trattare tra il 1306 e il 1310 ?

Anche su questo i documenti mi sembrano chiari. Per conto di Moroello Dante svolse missioni diplomatiche e "dettatorie" non solo rappresentandolo insieme ai suoi cugini nella vertenza con il vescovo di Luni che si concluse a Castelnuovo e forse anche -ma si tratta di un'ipotesi assai più congetturale, dato il suo ruolo di attore del negozio giuridico - suggerendo al notaio che la certificò di usare nell'arenga un passaggio di Cassiodoro, ${ }^{40} \mathrm{ma}$, poco prima o poco dopo, rispondendo in nome del marchese a un sonetto inviato da Cino da Pistoia..$^{41}$ La "pace di Castelnuovo" e Degno di voi trovar ogni tesoro, nella loro apparente diversità, rinviano infatti a un orizzonte comune, quello delle attività che svolgevano all'epoca di Dante gli "uomini di corte" - termine che merita una breve digressione.

Recentemente William Robins ha mostrato come Dante nella Commedia attribuisca un ruolo importante nel denunciare la corruzione della cortesia e dei costumi ad alcuni personaggi non aristocratici che in vita guidarono con le loro parole i potenti: Ciacco, Guglielmo Borsiere, Marco Lombardo. I primi commentatori, e tra loro i figli del poeta, definiscono unanimemente questi tre personaggi come "uomini di corte". ${ }^{42}$ Nella novella che dedica allo stesso Guglielmo Bor-

39 Per quanto il termine officium abbia indubbiamente il significato di 'dovere' che gli fornisce, specialmente quando accompagnato dal verbo "sequi", un valore giuridico in senso lato, la ripresa medievale di espressioni classiche come "officium liberalitatis" o "officium pietatis" mi pare dimostrare che esso non si configuri sistematicamente come dovere pubblico o "ufficiale".

40 Ha sostenuto la possibilità di una paternità dantesca Bertin, La pace di Castelnuovo Magra. Sulla base della comune citazione del passo di Cassiodoro tra questa pace e un'epistola inviata nel 1312 ad Enrico VII dalla corte di Cangrande, Pellegrini, Lo stilista nascosto, ha annunciato uno studio in cui attribuirà a Dante anche questa epistola.

41 Petrocchi, Vita di Dante, p. 100, afferma che «lo scambio di sonetti tra Cino (...) e Dante (...) non è necessariamente riconducibile a questo periodo, ché vige anche l'ipotesi d'una successiva permanenza di tutti e tre i corrispondenti alla corte di Enrico VII, al momento della presenza di Moroello durante la pacificazione di Vercelli», il che non muta di molto quanto qui sostenuto.

42 Robins, The case of courts entertainers. 
siere nel Decameron, Boccaccio che evidentemente ha in mente quanto scritto da Dante, nonché le parole dei suoi primi esegeti, spiega che anche costoro hanno subìto un processo di degenerazione: oggi, spiega, sparlano, seminano zizzania e fanno cattiverie, mentre, in precedenza,

soleva essere il lor mestiere e consumarsi la lor fatica in trattar paci, dove guerre o sdegni tra gentili uomini fosser nati, o trattar matrimonii, parentadi e amistà, e con belli motti e leggiadri ricreare gli animi degli affaticati e sollazzar le corti e con agre riprensioni, si come padri, mordere i difetti de' cattivi, e questo con premii assai leggieri. ${ }^{43}$

Seguendo una pista già tracciata di Michele Scherillo e Francesco Colagrosso, ${ }^{44}$ Robins ritiene che l'evocazione dantesca degli uomini di corte nella Commedia costituisca un momento in cui Dante riflette sul proprio ruolo di intellettuale in dialogo col potere. Certamente gli officia che i documenti attestano svolti da Dante presso i Malaspina assomigliano molto a quelli descritti da Boccaccio nel suo buon tempo antico.

La corte dei marchesi di Lunigiana, del resto, sin dal secolo XII aveva accolto uomini in cerca di fama accettando da loro, come attesta il corpus dei 36 testi trobadorici recentemente edito da Gilda Caiti-Russo, ${ }^{45}$ consigli e "sollazzi”. Considerando come in quel corpus tornino in nomi di Selvaggia e di Beatrice, sapendo che in quella corte era rinata una poesia politica in occitano alla fine del secolo XIII, la presenza di Dante presso un marchese che era nipote e omonimo di quello che nel 1273 era stato oggetto di un componimento di Luchetto Gattilusio $^{46}$ sembra acquistare uno spessore più ampio sia della mera necessità "cortigiana” di sopravvivere, sia, come si vedrà, della speranza di ottenere una mediazione per rientrare a Firenze. Il corpus dei testi danteschi plausibilmente composti negli anni 1306-1310 e le tracce lasciate dai Malaspina in luoghi strategici della sua riflessione successiva - canti “cortesi” della Commedia in testa mostrano infatti che il soggiorno presso Moroello costituì per Dante una fase importante di definizione delle proprie idee politiche e dei propri progetti letterari.

Forse si trattò di una fase più lunga di quello che normalmente si pensa. Come si è già ripetuto più volte il soggiorno di Dante in Lunigiana ha come termine ante quem la pace di Castelnuovo (6 ottobre 1306). Sulla scorta di una congettura basata su due elementi problematici e non datati (la testimonianza di Boccaccio nel Trattatello e il presunto "dittico" formato da epistola IV e

43 Boccaccio, Decameron, I giornata, novella VIII.

44 Scherillo, Ciacco e Dante; Colagrosso, Gli uomini di corte.

45 Caiti-Russo, Les troubadours.

46 Caiti-Russo, Les troubadours, p. 390; Luchetto Gattilusio, Liriche, p. 32. 
“montanina”) si colloca di solito la fine di questo soggiorno al 1307. ${ }^{47} \mathrm{Un}$ documento datato costituisce invece un forte indizio a favore dell'idea che due anni dopo il 1306 tale soggiorno proseguisse ancora - o fosse ripreso dopo un'interruzione - e porta dunque a sceglierlo come momento dopo il quale avvenne la sua interruzione definitiva. Si tratta della presenza, il 21 ottobre 1308 di Giovanni, primo figlio di Dante, a Lucca, città vicina geograficamente e politicamente a Moroello. ${ }^{48}$ Quanto poi tale soggiorno si protrasse non è possibile dire. Ma non essendo questa la sede per discutere delle altre tappe che gli studiosi attribuiscono a Dante in questi anni - se a Lucca il 31 marzo del 1309 una disposizione comunale stabilì che i fiorentini esuli dovessero allontanarsi dalla città nulla osta a ritenere che Dante poté rimanere in altre terre malaspiniane, controllate da Moroello, fino all'arrivo dell'imperatore e che proprio dalla corte di Moroello poté spostarsi verso quella di Enrico.

La ricerca degli ultimi anni ha chiarito come, dal punto di vista politico, per Dante il biennio 1306-1308 fu aperto (per effetto della perdita di Pistoia e Bologna, alleate dei Bianchi fiorentini) dal tramonto definitivo delle possibilità di vittoria del fronte in cui aveva militato nei primi anni dell'esilio e fu chiuso (per effetto forse della morte di Corso Donati il 6 ottobre 1308, più certamente del fallimento del tentativo di pacificazione di Napoleone Orsini, avvenuto qualche mese prima) con la fine della speranza di un suo ritorno pacifico a Firenze. Questo fatto ha spinto gli studiosi, da Umberto Carpi in poi, a collegare strettamente Moroello Malaspina e il progetto dantesco di tornare a Firenze non più con le armi, ma con la persuasione e il diritto, un progetto che sarebbe testimoniato dall'epistola perduta Popule mee. ${ }^{49}$

47 L'idea secondo cui Dante nell'epistola IV racconta del passaggio dalla Lunigiana al Casentino si basa sull'accostamento tra il riferimento alle montagne tra cui scorre l'Arno della canzone “montanina” («così m'ha' concio, Amore, in mezzo l'alpi / nella valle del fiume / lungo 'l qual sempre sopra me s'è forte») e quello al fiume Sarno (cioè Arno) dell'epistola IV che hanno fatto ritenere, anche alla luce del «sub fontes Sarni» dell'epistola VII, che si trattasse del Casentino. Boccaccio, spesso invocato, in realtà non fornisce argomenti dirimenti per confermare questo spostamento. La prima redazione del trattatello sembra effettivamente far riferimento a un soggiorno alternato tra Casentino e Lunigiana (e Montefeltro) compatibile con quel passaggio (Boccaccio, Trattatello, (I red.), par. 74: «egli [...] quando col conte Salvatico in Casentino, quando col Marchese Morruello Malespina in Lunigiana [...] si stette»). Ma la seconda redazione segnala solo un passaggio nel senso inverso (Boccaccio, Trattatello (II red.), par. 55: «quindi [da Verona] in Toscana tornatosene, per alcun tempo fu col conte Salvatico in Casentino. Di quindi fu col Marchese Morruello Malespina in Lunigiana»). Il fatto che il passaggio sia databile al 1307 (o al 1308) è una congettura basata sull'idea che per un po' di tempo in Lunigiana Dante dovette rimanere.

48 CDD, n. 98, pp. 153-154.

49 Carpi, Un inferno guelfo, pp. 26-34. Santagata, Dante, pp. 186-203. 
É possibile che le cose stiano così, ma al legame di questo testo con Moroello Malspina, tuttavia, e più in generale al fatto che il soggiorno di Dante in Lunigiana sarebbe stato marcato dal tentativo dantesco di rientrare a Firenze, si oppone un fatto importante che la ricerca recente non ha considerato. Dopo la conquista di Pistoia, nel 1306, Moroello cominciò infatti una lunga vertenza con Firenze in merito ai diritti di un palazzo pistoiese, quello appartenuto al pistoiese "bianco" Dino degli Ammannati. ${ }^{50}$ Il comune di Firenze considerava il palazzo confiscato bene di un bandito di una città di fatto soggetta, e per questo lo aveva assegnato al fiorentino Gherardo Tornaquinci. Il marchese, invece, riteneva fosse di sua proprietà sostenendo la sua famiglia che lo aveva acquistato da tempo e che Dino, al quale lo aveva concesso, ne deteneva solo il possesso, non la proprietà piena. Il conflitto legale scatenò contro Firenze l'ostilità di Moroello che, come scrive un erudito ottocentesco, «allora alquanto dispettò de' Guelfi». ${ }^{51}$ Attuando una rappresaglia, il Malaspina bloccò il passaggio dei fiorentini nelle sue terre e per alcuni anni li danneggiò notevolmente impedendo loro di raggiungere la strategica piazza commerciale di Genova. Solo nel 1309, spinta anche dalla necessità di riavvicinarsi ai Malaspina in funzione anti-pisana, ${ }^{52}$ Firenze cedette e il tribunale della Mercanzia, istituito l'anno precedente proprio per risolvere questioni relative alle rappresaglie, attribuì il palazzo a Moroello. Il meccanismo della rappresaglia, che rendeva i concittadini di un debitore responsabili del pagamento del debito, mostra che quello tra Firenze e Moroello era un conflitto che aveva importanti ripercussioni sul piano pubblico. Benché in quegli anni 1307-1309 i Malaspina e Firenze si trovassero a condividere in alcune occasioni alcuni obiettivi (come il progetto aragonese di conquista della Sardegna), Moroello si allontanò nettamente dall'alleanza con la città a cui era stato legato quando, comandando l'esercito lucchese, aveva condotto spedizioni e assedi. Firenze, peraltro, proprio in quegli anni vedeva riaprirsi conflitti interni in seguito alla spaccatura interna del fronte "nero" che ebbe come episodio culminante la morte di Corso Donati nel 1308.

Mi sembra dunque molto difficile ipotizzare che in queste circostanze Dante sperasse di ottenere dal Malaspina un appoggio per rientrare nella città con cui il marchese stava combattendo. Più probabile che vedesse nel soggiorno presso i Malaspina una vera alternativa, magari provvisoria, al ritorno in patria, e che dunque l'epistola Popule mee sia da ricondurre a una fase precedente, magari di poco, a quel soggiorno. Il fatto che l'unica fonte certa che ce ne parla, Leonar-

50 Riassunto e fonti in Salvadori, Malaspina, Moroello.

51 Gerini, Storia della Lunigiana, vol. I, p. 43.

52 Silva, Giacomo II d'Aragona. 
do Bruni, la collochi all'altezza del primo soggiorno veronese, dunque intorno al 1303-1304, spinge del resto nella stessa direzione. ${ }^{53}$

\section{Il piano della poesia. Il ritorno di Amore nel soggiorno in Lunigiana}

Alla luce di questi elementi, che concorrono a conferire al soggiorno lunigianese di Dante una durata più estesa e forse una diretta continuità con gli anni arrighiani, va riconsiderata anche la coeva produzione dantesca. L'immagine di Dante homo curialis, per quanto efficace, trova infatti il suo maggiore limite nel fatto che sin dal 1306 egli non scrisse solo per il marchese, ma anche per se stesso, anzi, in continuità con quanto aveva fatto sin dagli anni fiorentini, scrisse su se stesso.

Dopo l'esilio Dante aveva cominciato due progetti che in qualche modo costituivano forme di autolegittimazione e autopromozione, il De vulgari eloquentia e il Convivio. ${ }^{54}$ Com'è stato osservato più volte, in questi due lavori Dante cercò di costruire di sé un'immagine diversa da quella che poteva emergere dalla lettura della maggior parte delle Rime scritte sino a quel momento e della Vita Nova. Nel De vulgari eloquentia si presentò attraverso un'allusione appena velata come il massimo poeta della Virtù, lasciando a Cino da Pistoia il ruolo di campione della poesia d'Amore. Nel Convivio esplicitò sin dal principio il proposito di mondare sé stesso dalla possibile accusa di essere stato uno schiavo delle passioni procedendo a una monumentale reinterpretazione delle proprie canzoni in cui chiariva che quei testi dovevano essere letti come allegorie filosofiche.

Quando Dante nel 1306 arriva a Sarzana per stipulare la pace tra i Malaspina e il vescovo di Luni egli doveva avere con sé questi due lavori incominciati.

53 Appendice I. Le epistole perdute in Ep. (Baglio), pp. 228-245, p. 233.

54 Inglese, Vita di Dante, pp. 82-88 propone come inizio della stesura del De vulgari il 1304, e come termini per quella del IV libro del Convivio il marzo del 1306 (morte di Gherardo da Camino indicato come defunto in Conv. IV, xiv, 12) e il novembre 1308 (elezione di Enrico VII, di cui Dante è all'oscuro in Conv. IV, iii, 6). Tavoni, Qualche idea su Dante, p. 88, fornendo questi ed altri riferimenti conclude che i termini documentari indicano che il Convivio era stato iniziato tra nel 1303 o nel 1304 e interrotto tra marzo 1306 e gennaio 1309 (incoronazione di Enrico VII) e il De vulgari eloquentia iniziato nel 1304 e interrotto anch'esso entro il 1309. Questo autore, tuttavia, convinto della anteriorità dei due trattati alla trasformazione di Dante da filosofo laico in poeta-profeta, avvenuta a suo modo di vedere tra 1306-1307, ritiene probabile che i due trattati siano stati interrotti in coincidenza con quella trasformazione. 
Se non si hanno tracce che egli abbia proseguito il primo, è invece molto più probabile che per un po' continuò il secondo, per il quale, dopo aver potuto fruire delle biblioteche bolognesi, Dante si poté giovare forse di quelle, ben fornite, di Lucca. ${ }^{55} \mathrm{Ma}$, come si è accennato, a questi lavori si affiancano nuove produzioni scritte, che mostrano qualche differenza rispetto a quei progetti.

Una prima differenza è linguistica e si coglie nel modo in cui Dante, proseguendo con Cino una tenzone già avviata da qualche tempo, scrive Io sono stato con Amore insieme, che risponde al sonetto ciniano Dante, quando per caso s'abbandona. In questa occasione infatti Dante premette una epistola latina (la III) che fornisce la chiave per interpretare la composizione poetica. Come ha segnalato tra gli altri Anna Fontes Baratto, al principio del Convivio Dante aveva criticato la prassi di glossare testi volgari con testi latini. ${ }^{56}$ Dante sembra dunque modificare la sua opinione, introducendo un tipo di auto-commento che si distacca da quello progettato in precedenza. ${ }^{57}$ Molti interpreti, a cominciare da Contini, hanno avvicinato questa nuova modalità a una razo trobadorica. ${ }^{58}$ Considerando i rapporti di Cino con la corte malaspiniana, il ripensamento potrebbe essere collegato proprio a un primo contatto con questa corte che tuttavia, ancora una volta, è di difficile datazione (essendo l'ottobre del 1306, data della pace di Castelnuovo, solo il termine ante quem).

Dal momento che nell'intestazione dell'epistola III Dante chiama Cino «exulanti pistoriensi», essa non dovrebbe essere successiva all'11 aprile 1306, data della conquista nera di Pistoia (anche se Cino potrebbe essersi di nuovo allontanato dalla sua città). Si potrebbe dunque pensare che i contatti con la corte siano cominciati tra la primavera e l'estate di quell'anno, cioè nel corso dell'assedio di Pistoia. Se si volessero collegare strettamente l'epistola IV e la "montanina”, questa ipotesi permetterebbe di avvicinare cronologicamente e dal punto di vista dell'ambiente le due epistole che comunque, al di là di ogni dubbio, condividono non solo la funzione di accompagnamento di un testo volgare: una funzione che recentemente è stata definita da Natascia Tonelli come «un tratto fortemente congiuntivo», ${ }^{59}$ ma entro l'orizzonte socio-culturale con Cino mediatore tra Dante e il Marchese.

Un altro tratto che avvicina le due lettere è il contenuto. Tanto nel testo che l'epistola III introduce (Io sono stato con amore insieme), quanto nell'epistola IV

55 Introduzione in Conv. (Fioravanti), pp. 16-18.

56 Fontes Baratto, Le dyptique montanino, p. 66.

57 Anche se, come mi suggerisce Antonio Montefusco, sin dall'inizio dell'esilio il divieto del commento di poesie volgari con trattazioni latine ha comunque un macroscopico controesempio nel De Vulgari Eloquentia.

58 Commento in Rime (Contini), p. 206 (ma a proposito dell'epistola IV).

59 Tonelli, La canzone montanina, p. 10. 
Dante parla di sé come poeta d'amore e dice, in modi diversi, che amore è un signore al quale non si può resistere e al quale lui, personalmente, non resiste. Il ritorno di Dante alla poesia d'amore e alla definizione di sé come servo di Amore è graduale ma ineluttabile se osservato attraverso la prospettiva della corrispondenza con Cino, anche al di là delle due epistole. Per prudenza sarebbe meglio prescindere dai primi quattro sonetti (Novellamente Amor mi giura e dice, di Cino, con la risposta I'ho veduto già senza radice; e Perch'io non trovo chi meco ragioni con la risposta di Cino Dante, i’ non so il qual albergo soni) scambiati tra i due. Per alcuni studiosi, infatti, essi risalirebbero ancora al periodo precedente all'esilio. ${ }^{60}$ Se invece, come pensano altri, essi risalissero agli anni successivi al 1303 diventerebbe interessante osservare che se nel primo scambio Dante si ritaglia il ruolo di chi sconsiglia all'interlocutore di cedere a un nuovo amore, già nel secondo dichiara che il suo "gran disio (...) di dire $\mathrm{i}$ pensamenti boni» (vv. 3-4), in una sorta sublimazione del desiderio di poter parlare d'Amore, resa necessaria dalla mancanza di interlocutori nel luogo in cui si trova. Come si diceva, tuttavia, il ritorno all'amore di Dante si fa più chiaro con Io sono stato con Amore insieme, in cui si dice esplicitamente che la ragione non può nulla contro Amore («libero arbitrio già mai non fu franco / sì che consiglio invan vi si balestra», vv. 10-11), e trova una conferma nella risposta di Dante al sonetto Cercando di trovar minera in oro inviato da Cino a Moroello, dove afferma: «io che trafitto sono in ogni poro / del prun che con sospir si medicina» (vv. 5-6), senza far capire troppo se sta parlando davvero a nome del Marchese o non, piuttosto, a titolo personale.

Quest'autorappresentazione di Dante come servo e vittima di Amore stona con il proposito iniziale del Convivio ma trova il suo massimo compimento nell'epistola IV e nella canzone "montanina" che, se non altro, condividono immagini e figure di stile ad essa legate, al punto che, come si diceva presentando l'ipotesi Villa, nonostante l'assenza di prove ecdotiche molti interpreti hanno creduto e credono ancora che l'una sia stata scritta per accompagnare l'altra. Del resto, nel caso in cui si volesse aderire all'altra ipotesi, proposta di recente da Natascia Tonelli, secondo cui l'epistola IV nacque per accompagnare non quella sola quella canzone, ma l'intero "libro delle canzoni" allestito da Dante in quella fase, dal momento che quell'ordinamento avrebbe avuto come ultima canzone proprio la "montanina", ${ }^{61}$ il ritorno di Amore nell'autobiografia dantesca si configurerebbe in modo altrettanto forte. L'ipotesi di un "libro delle can-

60 È l'opinione che traspare dall'ordinamento del Barbi e che Contini, Rime, p. 137 riprende. Al contrario Pinto, La poetica dell'esilio, ritiene che anche questi sonetti risalgono al periodo successivo all'esilio.

61 Tonelli, Tre donne, p. 69; Tonelli, Rileggendo; Tonelli, La canzone montanina, p. 14. 
zoni” allestito da Dante, tuttavia, continua a dividere gli studiosi e queste pagine, per il loro tema e per le competenze di chi le scrive, non sono certo le più adatte per discuterla. Certo è che negli stessi anni in cui Dante, dentro o in prossimità della corte di Moroello, proseguiva la redazione di un'opera, il Convivio, nata dalla necessità di mondare il suo autore dalla macchia di essere stato schiavo delle passioni amorose, quelle passioni in modi diversi erano tornate a manifestarsi.

\section{Il piano della politica. Dante uomo di corte e d'impero?}

Quanto durarono quegli anni? Nonostante si legga quasi ovunque che il soggiorno in Lunigiana si concluse nel 1307 e che coincise col trasferimento in Casentino, in realtà, come si è visto, modi e ragioni della separazione sono assai meno certi. Certo, nell'epistola IV Dante afferma che da Moroello deve separarsi, anzi, la ragione principale dell'epistola sembra dettata proprio dalla necessità di giustificare quella separazione. Per quanto riguarda le cause della separazione, come si è accennato all'inizio, la critica si divide tra chi, privilegiando il senso letterale, ritiene che Dante stia parlando di un nuovo amore e chi, leggendola allegoricamente, adombra ragioni legate a una scrittura più o meno avanzata della Commedia. I documenti storici e lo studio del contesto che abbiamo preso in considerazione non permettono di confermare né la prima né la seconda ipotesi, ma, forse, con grande cautela, di dare al distacco da Moroello un senso leggermente diverso da quello che di solito gli si attribuisce.

Se infatti si accettano le proposte fatte nelle pagine precedenti, ovvero che la fase in cui Dante fu a contatto più o meno stretto con Moroello si prolungò almeno fino al 1308 (se non oltre), che essa non coincise per Dante con il progetto di rientrare a Firenze, che nell'ultima fase di quel periodo Dante poté trovare nel signore che lo proteggeva e nella sua corte, frequentata da Cino da Pistoia, un interlocutore con cui tanto tornare a parlare di Amore, quanto condividere la recente scoperta dell'Impero testimoniata dal "capitolo speziale” del IV libro del Convivio, allora il valore di cesura biografica del distacco descritto dall'epistola IV risulta molto diminuito. Negli anni successivi al 1308 infatti, Dante sarebbe restato fedele tanto all'idea di impero che aveva maturato allora, quanto all'idea del ritorno di Amore, ultimo esito della nuova riscrittura autobiografica con cui la fase dell'esclusività degli studi filosofici, da punto d'arrivo, diveniva in un certo senso una digressione conclusa, una parentesi, un "traviamento".

Se si rende il distacco dalla curia malaspiniana descritto dall'epistola IV meno drammatico e periodizzante, allora queste considerazioni rendono possi- 
bile avanzare con grandissima cautela una congettura sulla sua datazione e sul suo significato che recupera, per certi versi, la proposta di Claudia Villa.

L'epistola IV potrebbe infatti essere stata scritta non nel 1311-12, dopo l'incontro di Moroello (e probabilmente anche Dante) con Enrico VII, ma prima di quell'incontro, tra il momento dell'acquisizione da parte di Dante della notizia della morte di Alberto d'Asburgo, re dei romani mai sceso in Italia, o della elezione (novembre 1308) o della incoronazione a Re dei Romani (gennaio 1309) di Enrico VII e il raggiungimento dell'imperatore a Milano (fine 1310). ${ }^{62}$ In questo contesto di rinascita di una concreta speranza politica per l'Impero, già sognata nel IV trattato del Convivio, l'apparizione raccontata nella IV epistola potrebbe essere (anche?) un'allegoria, una rappresentazione, se non del ritorno di Beatrice, o dell'inizio della Commedia, almeno dell'avvento di una virtù come la giustizia imperiale, che nella fase precedente era stato possibile solamente rimpiangere, vagheggiandone la restaurazione in modi alternativi e assai più indiretti, che facevano leva (come nel De vulgari Eloquentia e nel IV trattato del Convivio) sulla memoria di Federico II e Manfredi e che invece adesso appariva lì a portata di mano, delineando un nuovo orizzonte di possibilità. Il riferimento all'apparizione di quella virtù avrebbe potuto giustificare un allontanamento di Dante da Moroello (per annunciare la notizia ad altri signori, magari proprio i Guidi casentinesi, per raggiungere gli alleati dell'imperatore, o l'imperatore stesso). Esprimere la necessità di partire per questa missione attraverso il vocabolario d'amore, lo stesso con il quale i due avevano, insieme a Cino, comunicato sin dal loro incontro, significava far leva su un orizzonte che come il tema della vera nobiltà Moroello avrebbe potuto condividere.

Gli elementi testuali a suffragare questa congettura non sono molti, ma sono significativi. Se in apparenza la donna descritta nell'epistola non sembra avere caratteristiche che rinviino in modo evidente alla giustizia o all'Impero, colpisce il doppio uso rilevato da Anna Fontes della similitudine della folgore che scende dal cielo in questa epistola e nella $\mathrm{V}$, dove descrive la sublimis aquila imperiale che sta per giungere in Italia e del verbo obstupescere usato qui per indicare la reazione di Dante all'apparizione, nella V, l'atteggiamento che il poeta auspica per gli italiani di fronte all'arrivo di Enrico. ${ }^{63} \mathrm{~A}$ una possibile lettura politica rinvia più in generale un'altra parte del testo, quella che presenta il nuovo avvento d'Amore come il ritorno di un bandito dopo un lungo esilio, il quale, finalmente trionfando, ha compiuto esattamente gli atti che compivano i signori fuoriusciti quando rientravano trionfatori: uccidere, espellere e imprigionare. Amore ha infatti, ucciso (occidit) il proposito di astenersi dalla poesia

62 Inglese, Vita di Dante, p. 99.

63 Fontes Baratto, L'Epistola V di Dante: un'intertestualità polimorfa, n. 30. 
d'amore; scacciato (expulit) le assidue meditazioni sulle cose terrene e celesti che ha - con vocabolario tecnicamente giudiziario - confinato come sospette («quasi suspecte impie relegavit», Ep. IV, 4), cioè fuor di metafora, messo da parte. Infine ha imprigionato (ligavit) il libero arbitrio paventando - con una scelta tratta sempre nello stesso campo semantico della politica - una "ribellione" dell'anima contro di lui. Anche se questi verbi potevano facilmente evocare quanto era avvenuto a Firenze nel 1302 quando a fare le spese del ritorno di Corso Donati era stato lo stesso Dante, oppure a Pistoia nel 1306, al termine di un assedio violentissimo compiuto da Moroello stesso, potrebbe trattarsi di evocazione di un ritorno imperiale che risuona, anticipandole, con le immagini di diversissima estrazione, ma di eguale violenza, delle lettere arrighiane, non solo della quinta.

Resta tuttavia aperto il problema del testo che questa epistola potrebbe aver accompagnato, il quale difficilmente in questi anni potrebbe essere il Purgatorio. Dunque, come si è detto, si tratta ancora di un'ipotesi. In conclusione, solo se si riuscisse a dimostrare che Dante all'altezza dell'epistola IV aveva già avuto notizia di Enrico VII, la possibilità che questi riferimenti siano stati pensati come accenni al ritorno di un signore diverso si farebbe più consistente. In attesa di tale dimostrazione è più prudente, anche in questo caso, sospendere il giudizio.

\section{Per una conclusione: gradualità e catastrofe nel percorso dantesco}

Anche al di là di quest'ultima possibilità, anche volendo ritenere che questa lettera sia stata scritta prima della notizia del possibile arrivo dell'imperatore nel contesto italiano, la sua lettura secondo la prospettiva del destinatario fa comprendere come negli anni dell'incontro con la curia di Moroello Dante abbia modificato i suoi piani. Mentre era ancora impegnato nell'autoanalisi moralizzatrice del Convivio sorta, come ci attesta lui stesso, dal bisogno di mondare la propria fama e tornare in patria, ricominciò, grazie agli invii di Cino e in virtù della (ri)scoperta di una tradizione culturale cortese, a scrivere d'Amore e finì per dichiararsi, con la "montanina" e/o l'epistola IV, a quello del tutto vincolato e - anche per questo ${ }^{64}$ - ormai impossibilitato a tornare in patria.

Come abbiamo ripetuto, il racconto dell'epistola, nel suo lapidario riferirsi a un ordine a cui è impossibile disobbedire, non permette attualmente di identi-

64 Carpi, Un congedo da Firenze. 
ficare con certezza quali possano esser state le "vere" ragioni che lo portarono a partire dalla corte lunigianese. La proposta avanzata nel paragrafo precedente resta una congettura da prendere con il beneficio di inventario. Anche al di là di tale congettura, tuttavia, resta l'opportunità di considerare insieme i piani della politica e quelli della poesia. La scelta tra questi due piani, che divide gli interpreti talvolta sovrapponendosi a quella tra interpretazione allegorica e letterale di questo testo, può essere infatti, un falso problema. Più probabile, mi pare, che al graduale cambiamento di prospettiva che quel testo mette in scena in modo drammatico contribuissero tanto la riflessione politica quanto quella poetica: tanto l'evolversi del progetto tratteggiato nel De Vulgari Eloquentia di trovare un modo per supplire, con la lingua e la poesia, alla dispersione della "curia italiana" quanto le sperimentazioni linguistica e letteraria utili a tale supplenza; tanto l'uso delle proprie canzoni come mezzo per comunicare con membri della nobiltà italiana, quanto l'enciclopedia allestita nella forma del loro commento che aveva finito per lasciare uno spazio smisurato alla riflessione sull'impero. Tutte queste linee di ricerca trovarono nella curia di Moroello, membro di una dinastia che vantava una tradizione illustre di esercizio pubblici poteri e una lunga frequentazione con i trovatori, un laboratorio ideale.

Che il commiato grato da quella corte raccontato da Dante nell'epistola IV costituisca un duplice passo, politico e poetico, verso l'orizzonte della Commedia mi pare altamente plausibile. Sul piano politico lo testimonia il fatto che, nel licenziare Inferno e Purgatorio a qualche anno di distanza dall'incontro con quella curia, Dante non rinnegò affatto l'amicizia coi Malaspina. Sul piano poetico lo dimostra la rivendicazione in quelle cantiche di un'idea di cortesia e di amore a cui proprio in Lunigiana Dante aveva deciso di (tornare ad) aderire. Questa lunga fedeltà, non così frequente in Dante, conferma nell'idea, presupposta nelle pagine che precedono, di leggere l'epistola IV non come un addio definitivo dal suo destinatario, come talvolta è stato fatto, ma piuttosto, come una mossa volta a mantenere il rapporto aperto, nella continuità di un'adesione a valori comuni. Ancora da dimostrare è tuttavia che quel passo verso la Commedia fu il solo o definitivo. Il ritorno di Amore potrebbe aver costituito per Dante un cambiamento di prospettiva necessario ma non sufficiente per dedicarsi completamente al lavoro sulla sua opera maggiore.

Raramente, del resto, le svolte effettivamente avvenute nella biografia dantesca coincidono perfettamente con quelle raccontate nell'autobiografia. E questo è spiegabile con la differenza tra processi graduali e catastrofi. Tanto nel periodo precedente quanto in quello successivo al soggiorno in Lunigiana, Dante prese alcuni passaggi del suo passato che avevano costituito il frutto di elaborazioni meditate e protratte (la vocazione alla scrittura poetica, lo studio della filosofia, i rapporti più o meno buoni con gli altri Bianchi, l'adesione all’Impero) 
e li drammatizzò, al fine di conferire loro senso e poterli raccontare, mettendoli in scena come eventi istantanei e puntiformi (l'incontro con Beatrice, la sua morte, l'abbandono della «compagnia malvagia e scempia», l’illuminazione sul ruolo provvidenziale di Roma).

In questa serie, credo, si può inserire anche la folgorazione lungo il corso dell'Arno raccontata nell'epistola IV, dietro la quale occorre forse riconoscere in realtà il punto di arrivo di un processo di adattamento levigato dal ragionamento, perché, se il mutare delle circostanze esistenziali costituì per Dante, quasi sempre un problema - un problema non solo individuale, ma anche collettivo e universale - la creazione poetica, e più generalmente la scrittura, furono sistematicamente il mezzo per trovare una - altrettanto individuale, collettiva e universale - soluzione. 